\title{
Texture-Based Classification of Atherosclerotic Carotid Plaques
}

\author{
C. I. Christodoulou*, C. S. Pattichis, Member, IEEE, M. Pantziaris, and A. Nicolaides
}

\begin{abstract}
There are indications that the morphology of atherosclerotic carotid plaques, obtained by high-resolution ultrasound imaging, has prognostic implications. The objective of this study was to develop a computer-aided system that will facilitate the characterization of carotid plaques for the identification of individuals with asymptomatic carotid stenosis at risk of stroke. A total of 230 plaque images were collected which were classified into two types: symptomatic because of ipsilateral hemispheric symptoms, or asymptomatic because they were not connected with ipsilateral hemispheric events. Ten different texture feature sets were extracted from the manually segmented plaque images using the following algorithms: first-order statistics, spatial gray level dependence matrices, gray level difference statistics, neighborhood gray tone difference matrix, statistical feature matrix, Laws texture energy measures, fractal dimension texture analysis, Fourier power spectrum and shape parameters. For the classification task a modular neural network composed of self-organizing map (SOM) classifiers, and combining techniques based on a confidence measure were used. Combining the classification results of the ten SOM classifiers inputted with the ten feature sets improved the classification rate of the individual classifiers, reaching an average diagnostic yield (DY) of $73.1 \%$. The same modular system was implemented using the statistical k-nearest neighbor (KNN) classifier. The combined DY for the KNN system was $68.8 \%$. The results of this paper show that it is possible to identify a group of patients at risk of stroke based on texture features extracted from ultrasound images of carotid plaques. This group of patients may benefit from a carotid endarterectomy whereas other patients may be spared from an unnecessary operation.
\end{abstract}

Index Terms-Carotid plaques, self-organizing map, texture, ultrasound imaging.

\section{INTRODUCTION}

$\mathbf{T}$ HERE is evidence that carotid endarterectomy in patients with asymptomatic carotid stenosis will reduce the incidence of stroke [1]. However, a large number of patients may be operated unnecessarily. Therefore, it is necessary

Manuscript received October 2, 2002; revised January 2, 2003. This paper was supported by a grant from the Government of Cyprus, Planning Bureau. The Associate Editor responsible for coordinating the review of this paper and recommending its publication was W. Niessen. Asterisk indicates corresponding author.

*C. I. Christodoulou is with the Cyprus Institute of Neurology and Genetics, P.O. Box 3462, 1683 Nicosia, Cyprus (e-mail: cschr2@ucy.ac.cy).

C. S. Pattichis is with the Department of Computer Science, University of Cyprus, 1678 Nicosia, Cyprus (e-mail: pattichi@ucy.ac.cy).

M. Pantziaris is with the Cyprus Institute of Neurology and Genetics, 1683 Nicosia, Cyprus (e-mail: pantziari@ucy.ac.cy).

A. Nicolaides is with the Cyprus Institute of Neurology and Genetics, 1683 Nicosia, Cyprus (e-mail: anicolai@ucy.ac.cy) and with the Irvine Laboratory for Cardiovascular Investigation and Research, St Mary's Hospital Medical School, Imperial College of Science, Technology and Medicine, London W2 1PG, U.K. Digital Object Identifier 10.1109/TMI.2003.815066 to identify patients at high risk, which will be considered for carotid endarterectomy, and patients at low risk, which should be spared from an unnecessary, expensive and often dangerous operation. There are indications that the morphology of atherosclerotic carotid plaques, obtained by high-resolution ultrasound imaging, has prognostic implications [2]-[7]. Smooth surface, echogenicity and a homogenous texture are characteristics of stable plaques, whereas irregular surface, echolucency and a heterogeneous texture are characteristics of potentially unstable plaques. The objective of this study was to develop a computer-aided system that will facilitate the characterization of carotid plaques for the identification of individuals with asymptomatic carotid stenosis at risk of stroke.

In previous work, Geroulakos et al. [2] tested the hypothesis that the ultrasonic characteristics of carotid artery plaques were closely related to symptoms, and that the plaque structure may be an important factor in producing stroke, perhaps more than the degree of stenosis. An association was found of echolucent plaques with symptoms and cerebral infractions, which provided further evidence that echolucent plaques are unstable and tend to embolize. El-Barghouty et al. [3] in a study with 94 plaques reported an association between carotid plaque echolucency and the incidence of cerebral computed tomography brain infractions. The gray scale median (GSM) of the ultrasound plaque image was used for the characterization of plaques as echolucent (GSM $\leq 32$ ) and echogenic (GSM > 32). Iannuzzi et al. [4] analyzed 242 stroke and 336 transient ischemic attack (TIA) patients and identified significant relationships between carotid artery ultrasound plaque characteristics and ischemic cerebrovascular events. The features that were more consistently associated with TIAs were low echogenicity of carotid plaques, thicker plaques, and presence of longitudinal motion. Elatrozy et al. [5] in a study where 80 patients were examined, reported that plaques with GSM $<40$ are more related to ipsilateral hemispheric symptoms. Wilhjelm et al. [6] in a study with 52 patients scheduled for endarterectomy, presented a quantitative comparison between subjective classification of the ultrasound images, first- and second-order statistical features, and a histological analysis of the surgically removed plaque. Some correlation was found between the three types of information where the best performing feature was found to be the contrast. Finally, Polak et al. [7] in a study where 4886 individuals were followed up for an average of 3.3 years, found that hypoechoic carotid plaques, as seen on ultrasound images of the carotid arteries, are associated with increased risk of stroke. The plaques were manually categorized as hypoechoic, isoechoic or hyperechoic by independent readers. Polak et al. [7] further suggested that the subjectively grading of the plaque characteristics 
THE CAROTID PLAQUE MULTI-FEATURE MULTI-CLASSIFIER SYSTEM

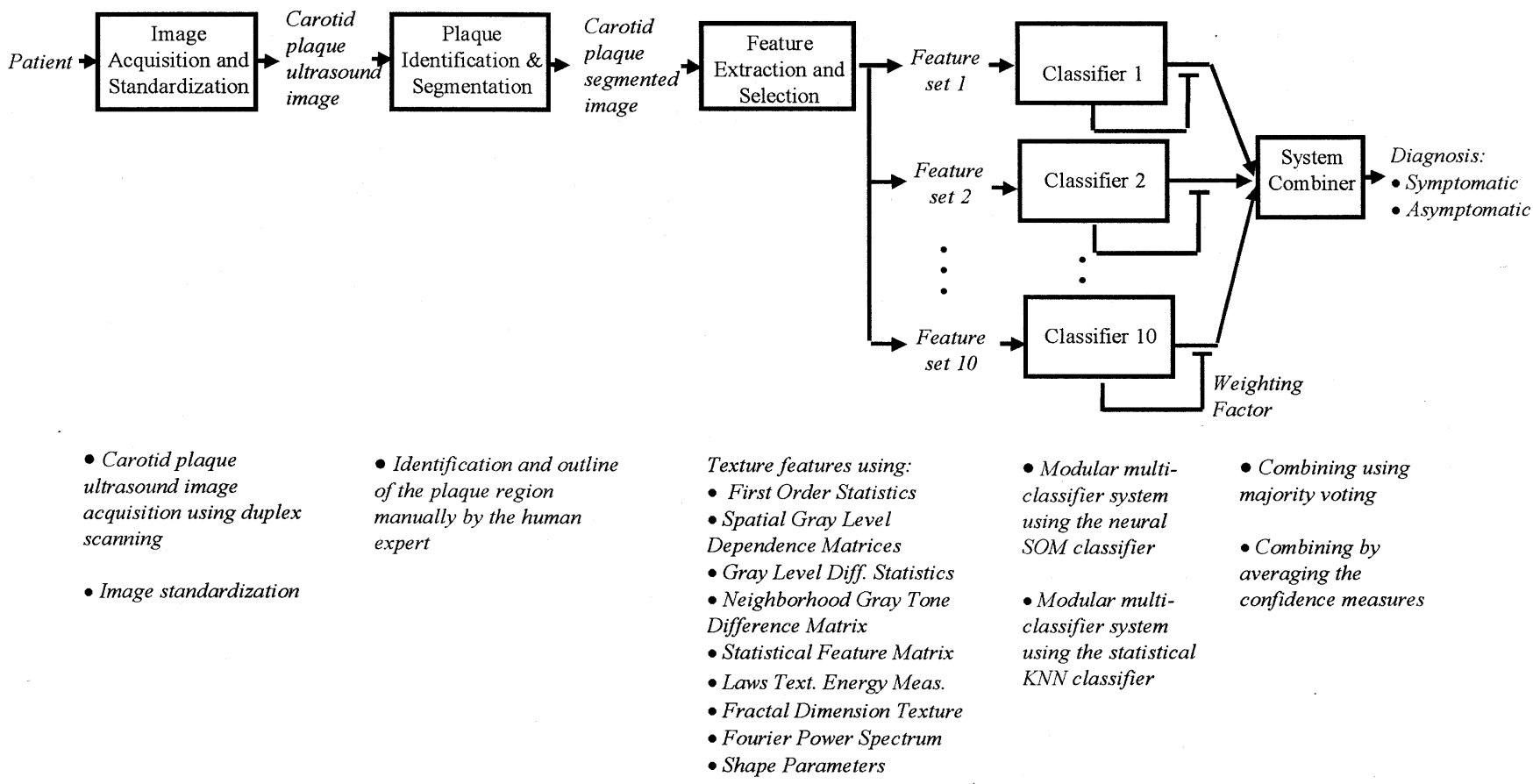

Fig. 1. Flowchart of the carotid plaque classification system based on a multifeature multiclassifier methodology.

might be improved by the use of quantitative methods, as proposed in this study.

The main objective of this study was to develop a computer-aided system using multifeature texture analysis, neural network classifiers and statistical pattern recognition techniques, for the automated characterization of carotid plaques recorded from high-resolution ultrasound images. The computer-aided classification of carotid plaques will contribute toward a more standardized and accurate methodology for the assessment of carotid plaques. The developed system should be able, based on extracted texture feature and shape parameters, to automatically classify plaques into one of the following types: 1) symptomatic because of ipsilateral hemispheric symptoms; 2) asymptomatic because they were not connected with ipsilateral hemispheric events. The aim is to identify patients at risk of stroke. The system is composed from the following modules: 1) image acquisition and preprocessing; 2) feature extraction; 3) plaque classification; and 4) system combiner. Fig. 1 illustrates the flowchart of the system.

\section{MATERIAL}

A total of 230 carotid plaque ultrasound images (115 symptomatic and 115 asymptomatic) obtained from 209 subjects, were analyzed. For some subjects plaques were obtained from both the right and left carotid arteries. Two sets of data were selected: 1) for training the system; 2) for evaluating its performance. For training the system, 80 symptomatic and 80 asymptomatic plaques were used, whereas for evaluation of the system, the remaining 35 symptomatic and 35 asymptomatic plaques were used. Five different bootstrap sets were constructed, where in each set $160(80+80)$ plaques were selected at random for training and $70(35+35)$ plaques for evaluation. The carotid plaques were labeled as symptomatic after one of the following symptoms was identified: stroke, TIA, or amaurosis fugax (AF). Hemorrhagic plaques, whose structure might change after the incidence, were excluded from the set of plaques under investigation. The aim of the system was to identify plaques with the potential to generate an incidence (stroke, TIA or AF).

\section{IMAGE ACQUisition AND PREPROCESSING}

The ultrasound images were collected at the Irvine Laboratory for Cardiovascular Investigation and Research, Saint Marys Hospital, U.K., by two ultrasonographers using an ATL (model HDI 3000; Advanced Technology Laboratories, Leichworth, U.K.) duplex scanner with a 7-4 MHz multifrequency probe. Longitudinal scans were performed using duplex scanning and color flow imaging [8]. B-mode scan settings were adjusted so that the maximum dynamic range was used with a linear postprocessing curve. The position of the probe was adjusted so that the ultrasonic beam was vertical to the artery wall. The time gain compensation curve was adjusted (gently sloping) to produce uniform intensity of echoes on the screen, but it was vertical in the lumen of the artery where attenuation in blood was minimal so that echogenicity of the far wall was the same as that of the near wall. The overall gain was set so that the appearance of the plaque was assessed to be optimal and noise appeared within the lumen. It was then decreased so that at least some areas in the lumen appeared to be free of noise (black) [8]. The resolution of the images was in the order of $700 \times 500$ pixels and the average size and standard deviation of the segmented images was $351 \pm 104 \times 100 \pm 32$ pixels. 


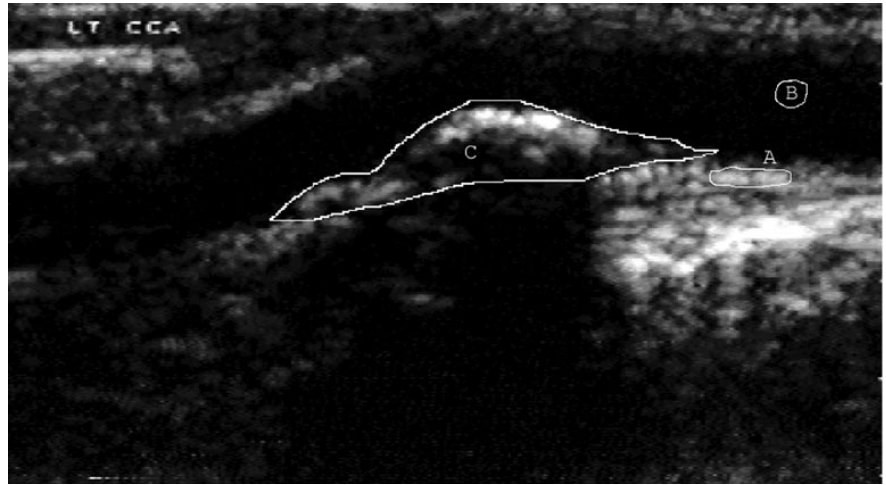

Fig. 2. An ultrasound image of the carotid artery with the outline of the carotid plaque (C), and the outlines of the adventitia (A) and blood (B) regions that were used as reference points for the image standardization.

Before processing, the images were standardized manually by linearly adjusting the image so that the median gray level value of the blood was 15-20, and the median gray level value of the adventitia (artery wall) was 180-200. The scale of the gray level of the images ranged from 0 to 255 . This standardization using blood and adventitia as reference points was necessary in order to extract comparable measurements in case of processing images obtained by different operators or different equipment [8]. Following the image standardization, the expert physician using as guide their corresponding color blood flow images segmented the plaques manually. Fig. 2 shows an ultrasound image with the plaque region outlined, as well as the adventitia and blood regions used as reference points for the standardization. Fig. 3 shows some examples of segmented symptomatic and asymptomatic plaques.

\section{FEATURE EXTRACTION}

Texture features and shape parameters were extracted from the segmented plaque images in order to be used for the classification of the carotid plaques. Texture contains important information that is used by humans for the interpretation and the analysis of many types of images. Texture refers to the spatial interrelationships and arrangement of the basic elements of an image [9]. Visually, these spatial interrelationships and arrangements of the image pixels are seen as variations in the intensity patterns or gray tones. Therefore, texture features have to be derived from the gray tones of the image. Although it is easy for humans to recognize texture, it is quite a difficult task to be defined, and subsequently to be interpreted by digital computers.

In this study, a total number of 61 texture features and shape parameters were extracted from the plaque segments using the following algorithms. The tested feature sets were also successfully used in previous work in texture analysis [10]-[13]. Some of the used features capture complementary textural properties, however, features that were highly dependent or similar with features in other feature sets, were identified through statistical analysis and eliminated. The implementation details for the texture algorithms can be found in the referred papers.

\section{A. First-Order Statistics (FOS)}

The following statistical features were computed [14]:
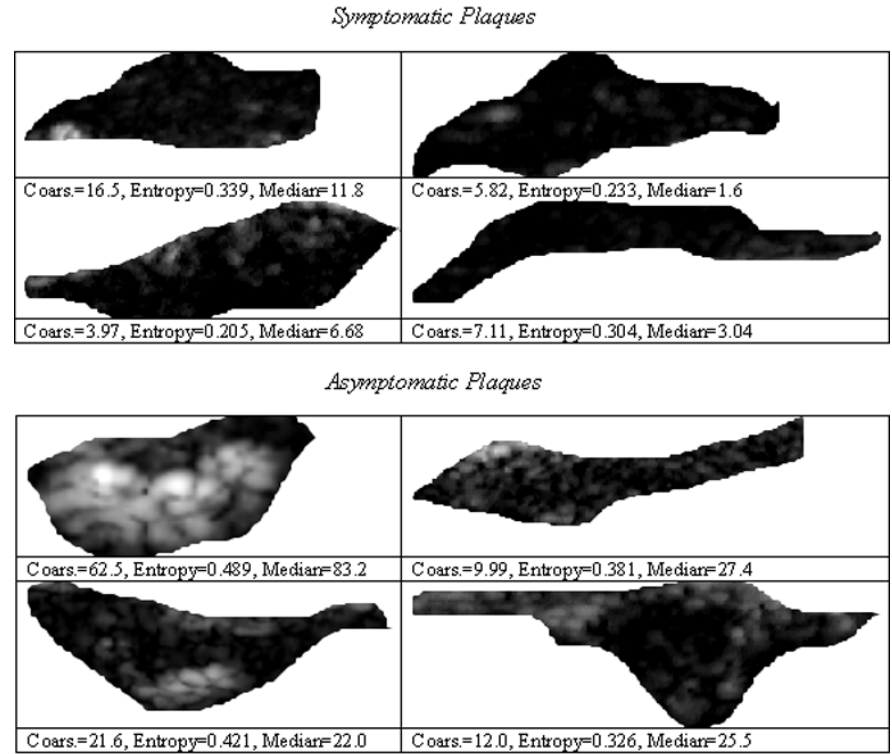

Fig. 3. Examples of symptomatic and asymptomatic plaques as these were segmented by the expert physician. Selected texture values are given for the features Coarseness (36), Entropy (27) and Median (2). The numbers in brackets denote the serial feature number as listed in Table I.

1) mean value, 2) median value, 3) standard deviation, 4) skewness, and 5) kurtosis.

\section{B. Spatial Gray Level Dependence Matrices (SGLDM)}

The SGLDM as proposed by Haralick et al. [15] are based on the estimation of the second-order joint conditional probability density functions (pdfs) that two pixels $(k, l)$ and $(m, n)$ with distance $d$ in direction specified by the angle $\theta$, have intensities of gray level $i$ and gray level $j$. Based on the pdfs the following texture measures [15] were computed:

1) Angular second moment, 2) Contrast, 3) Correlation, 4) Sum of squares: variance, 5) Inverse difference moment, 6) Sum average, 7) Sum variance, 8) Sum entropy, 9) Entropy, 10) Difference variance, 11) Difference entropy, and 12), 13) Information measures of correlation.

For a chosen distance $d$ (in this paper $d=1$ was used, i.e., $3 \times 3$ matrices) and for angles $\theta=0^{\circ}, 45^{\circ}, 90^{\circ}$ and $135^{\circ}$ we computed four values for each of the above 13 texture measures. In this paper, the mean and the range of these four values were computed for each feature, and they were used as two different feature sets.

\section{Gray Level Difference Statistics (GLDS)}

The GLDS algorithm [10] uses first-order statistics of local property values based on absolute differences between pairs of gray levels or of average gray levels in order to extract the following texture measures:

1) Contrast, 2) angular second moment, 3) entropy, and 4) mean.

The above features were calculated for displacements $\delta=$ $(0,1),(1,1),(1,0),(1,-1)$, where $\delta \equiv(\Delta x, \Delta y)$, and their mean values were taken. 


\section{Neighborhood Gray Tone Difference Matrix (NGTDM)}

Amadasun and King [9] proposed the NGTDM in order to extract textural features, which correspond to visual properties of texture. The following features were extracted, for a neighborhood size of $3 \times 3$ :

1) Coarseness, 2) contrast, 3) busyness, 4) complexity, and 5) strength.

\section{E. Statistical Feature Matrix (SFM)}

The SFM [16] measures the statistical properties of pixel pairs at several distances within an image, which are used for statistical analysis. Based on the SFM the following texture features were computed:

1) Coarseness, 2) contrast, 3) periodicity, and 4) roughness.

The constants $L_{r}, L_{c}$ which determine the maximum intersample spacing distance were set in this paper to $L_{r}=L_{c}=4$.

\section{F. Laws Texture Energy Measures (TEM)}

For the laws TEM extraction [11], [17], vectors of length $l=$ $7, L=(1,6,15,20,15,6,1), E=(-1-4,-5,0,5,4,1)$, and $S=(-1-2,1,4,1-2-1)$ were used, where $L$ performs local averaging, $E$ acts as edge detector and $S$ acts as spot detector. If we multiply the column vectors of length $l$ by row vectors of the same length, we obtain Laws $l \times l$ masks. In order to extract texture features from an image, these masks are convoluted with the image and the statistics (e.g., energy) of the resulting image are used to describe texture. The following texture features were extracted.

1) LL-texture energy from LL kernel; 2) EE-texture energy from EE kernel; 3) SS-texture energy from SS kernel; 4) LE-average texture energy from LE and EL kernels; 5) ES-average texture energy from ES and SE kernels; and 6) LS-average texture energy from LS and SL kernels.

\section{G. Fractal Dimension Texture Analysis (FDTA)}

Mandelbrot [18] developed the fractional Brownian motion model in order to describe the roughness of natural surfaces. The Hurst coefficient $H^{(k)}$ [11] was computed for image resolutions $k=1,2,3,4$. A smooth surface is described by a large value of the parameter $H$ whereas the reverse applies for a rough surface.

\section{H. Fourier Power Spectrum (FPS)}

The radial sum and the angular sum of the discrete Fourier transform [10] were computed in order to describe texture.

\section{Shape Parameters}

The following shape parameters were calculated from the segmented plaque image.

1) $\mathrm{X}$-coordinate maximum length;2) $\mathrm{Y}$-coordinate maximum length; 3 ) area; 4) perimeter; and 5) perimeter ${ }^{2} /$ area.

A simple figure of merit for the discriminatory value of each individual feature could be defined by computing the distance between the two classes for each feature as follows:

$$
\text { dis }=\frac{\left|m_{1}-m_{2}\right|}{\sqrt{\sigma_{1}^{2}+\sigma_{2}^{2}}}
$$

where $m_{1}$ and $m_{2}$ are the mean values and $\sigma_{1}$ and $\sigma_{2}$ are the standard deviations of the two classes for each feature. Best features are considered to be the ones with the greatest distance. More elaborate feature selection techniques, like sequential feature forward and backward selection [19], were also tested in order to identify the best features. However, when the selected best features were used as a separate feature set they performed worse than the individual feature sets, or the best features selected with the simple process using (1). This can be attributed to the high degree of overlap between the two classes, which did not allow a reliable estimate of the best feature combinations. Future work will investigate the use of more efficient feature selection techniques, like floating search [19], which may provide a better identification of the best features.

\section{Plaque Classification}

Following the feature extraction, feature classification was implemented based on a multifeature multiclassifier modular architecture [20]. For the classification of the carotid plaques into symptomatic or asymptomatic two different classifiers were used: 1) the neural network self-organizing map (SOM) classifier [21] and 2) the statistical K-nearest neighbor (KNN) classifier. Each one of the ten features sets described in the previous section, was used as input to a classifier and the results were combined. All features were normalized before use by subtracting their mean value and dividing with their standard deviation

$$
n\left(x_{i}\right)=\frac{\left(x_{i}-m_{i}\right)}{\sigma_{i}}
$$

where $m_{i}$ is the mean value and $\sigma_{i}$ is the standard deviation of the feature $i$.

\section{A. The SOM Classifier}

The SOM classifier is an unsupervised learning algorithm where the input patterns are freely distributed over the output node matrix [21]. The weight vectors of the output nodes are adapted without supervision in such a way, so that the density distribution of the input data is preserved and represented on the output nodes. This mapping of similar input patterns to output nodes, which are close to each other, represents a discretization of the input space, allowing a visualization of the distribution of the input data (see Fig. 4). The output nodes are usually ordered in a two-dimensional (2-D) grid and at the end of the training phase, the output nodes are labeled with the number of the input patterns per class, assigned to each node.

In the evaluation phase, a new input pattern was assigned to the winning output node with the weight vector closest to the new input vector. In order to classify the new input pattern, the majority of the labels of the output nodes in a neighborhood window (e.g., $3 \times 3$ ) centered at the winning node, were considered. The number of the input patterns in the neighborhood window for the two classes $m=\{1,2\},(1=$ symptomatic, $2=$ asymptomatic), was computed as

$$
S N_{m}=\sum_{i=1}^{L} W_{i} N_{m i}
$$




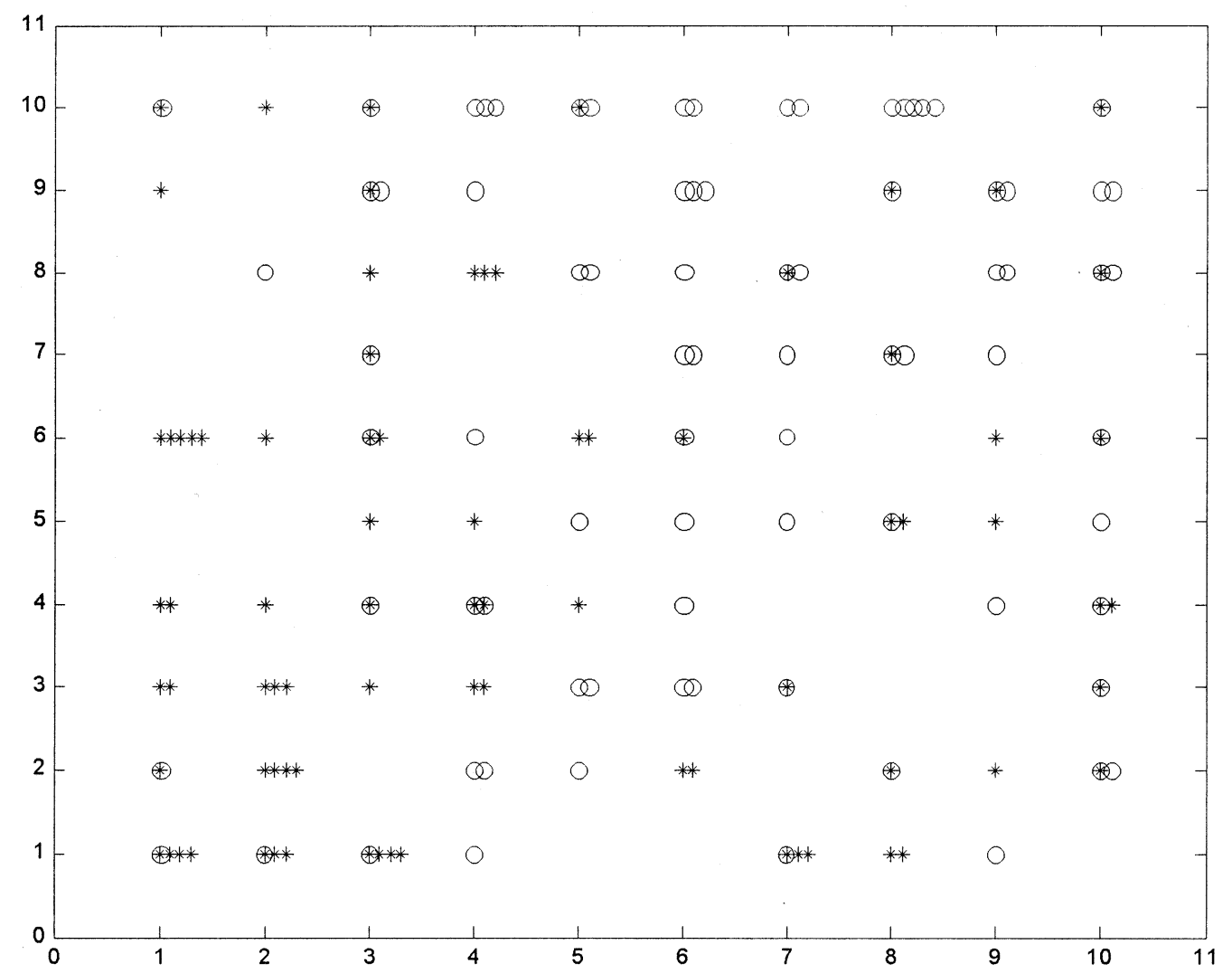

Fig. 4. Distribution of 160 carotid plaques of the training set ( 80 symptomatic and 80 asymptomatic) on a $10 \times 10$ SOM using as input the SGLDM (range of values) feature set which gave in average the highest DY $(*=$ symptomatic, $o=$ asymptomatic). Similar plaques are assigned to neighboring output matrix nodes. A new plaque will be assigned to one winning output node and will be classified based on the labels of the neighboring nodes in a $5 \times 5$ neighborhood window. The output nodes near to the winning node are given a greater weight than the ones farther away.

where $L$ is the number of the output nodes in the $R \times R$ neighborhood window with $L=R^{2}$ (e.g., $L=9$ using a $3 \times 3$ window), and $N_{m i}$ is the number of the training patterns of the class $m$ assigned to the output node $i$. $W_{i}=1 /\left(2 d_{i}\right)$, is a weighting factor based on the distance $d_{i}$ of the output node $i$ to the winning output node. $W_{i}$ gives the output nodes near to the winning output node a greater weight than the ones farther away (e.g., in a $3 \times 3$ window, for the winning node $W_{i}=1$, for the four nodes perpendicular to the winning node $W_{i}=0.5$ and for the four nodes diagonally located $W_{i}=0.3536$, etc). The evaluation input pattern was classified to the class $m$ of the $S N_{m}$ with the greatest value, as symptomatic or asymptomatic.

Beyond the classification result, a confidence measure on how reliable the classification result was computed as

$$
\text { conf }=2\left(\frac{\max \left\{S N_{1}, S N_{2}\right\}}{\left(S N_{1}+S N_{2}\right)}\right)-1
$$

If the plaque was classified as symptomatic then the computed confidence measure was multiplied with -1 . The confidence measure has a range of values from -1 (symptomatic) to 1 (asymptomatic). Values close to zero mean low confidence of the correctness of the classification result whereas values close to -1 or 1 indicate a high confidence. Fig. 4 shows the distribution of the 160 carotid plaques of the training set on a $10 \times 10$ SOM using as input the SGLDM (range of values) feature set.

\section{B. The KNN Classifier}

For comparison reasons, the statistical KNN classifier was also used for the classification of the carotid plaques. In the $\mathrm{KNN}$ algorithm, in order to classify a new input pattern, its $k$ nearest neighbors from the training set are identified [22]. The new pattern is classified to the most frequent class among its neighbors based on a similarity measure that is usually the Euclidean distance. In this paper, the KNN carotid plaque classification system was implemented for different values of $k=1,3$, 5,7 , and 9 , and it was tested using for input the ten different feature sets. In the case of the KNN, the confidence measure was simply computed as given in (4) with $S N_{m}$ being the number of the nearest neighbors per class $m$.

\section{Combining Classifiers}

In the case of difficult pattern recognition problems, the combination of the outputs of multiple classifiers using for input multiple feature sets extracted from the raw data, can improve the overall classification performance [23], [24]. The combination of the classification results of the different features and the different classifiers increases the probability that the errors of the individual features or classifiers may be compensated by the correct results of the rest. The use of a confidence measure by weighting the individual classification results before combining can further improve the overall performance. However, it should be noted that combining techniques would perform optimally 
TABLE I

Statistical ANALysis of the 15 Best TeXture Features Computed From 230 (115 Symptomatic and 115 Asymptomatic) Ultrasound IMAGES OF Carotid Atherosclerotic Plaques. For Each Feature the Mean and Standard Deviation Were Computed For the Symptomatic Group and for the Asymptomatic Group. The Distance Between the Symptomatic and the Asymptomatic Groups Was Computed as Described IN (1) AND the FEATURes Were AsSigned a RANK ORDER ACCORding to THEIR INTERCLASS Distance

\begin{tabular}{|c|c|c|c|c|c|c|c|}
\hline \multirow[b]{2}{*}{ No } & \multirow[b]{2}{*}{ Texture Feature } & \multicolumn{2}{|c|}{ Symptomatic } & \multicolumn{2}{|c|}{ Asymptomatic } & \multirow{2}{*}{$\begin{array}{l}\text { Distance } \\
d i s=\frac{\left|m_{1}-m_{2}\right|}{\sqrt{\sigma_{1}^{2}+\sigma_{2}^{2}}}\end{array}$} & \multirow[b]{2}{*}{$\begin{array}{l}\text { Rank } \\
\text { Order }\end{array}$} \\
\hline & & $\begin{array}{c}\text { Mean } \\
m_{1}\end{array}$ & $\begin{array}{c}\text { Std } \\
\sigma_{I}\end{array}$ & $\begin{array}{c}\text { Mean } \\
\mathrm{m}_{2}\end{array}$ & $\begin{array}{c}\text { Std } \\
\sigma_{2}\end{array}$ & & \\
\hline \multicolumn{8}{|c|}{ First Order Statistics (FOS) } \\
\hline 2 & Median & 15.71 & 16.62 & 29.40 & 22.87 & 0.484 & 10 \\
\hline \multicolumn{8}{|c|}{ Spatial Gray Level Dependence Matrices (SGLDM) - Mean values } \\
\hline 6 & Angular sec. moment & 0.166 & 0.187 & 0.065 & 0.120 & 0.456 & 11 \\
\hline 10 & Inverse diff. moment & 0.485 & 0.183 & 0.354 & 0.161 & 0.538 & 6 \\
\hline 11 & Sum average & 57.09 & 33.67 & 82.67 & 44.95 & 0.455 & 12 \\
\hline 13 & Sum entropy & 3.76 & 1.16 & 4.62 & 1.00 & 0.560 & 5 \\
\hline 14 & Entropy & 4.73 & 1.62 & 5.97 & 1.45 & 0.570 & 4 \\
\hline 15 & Difference variance & 280.5 & 119.8 & 219.7 & 65.8 & 0.445 & 15 \\
\hline \multicolumn{8}{|c|}{ Spatial Gray Level Dependence Matrices (SGLDM) - Range of values } \\
\hline 19 & $\begin{array}{l}\text { Angular second } \\
\text { moment }\end{array}$ & 0.0095 & 0.0055 & 0.0050 & 0.0050 & 0.610 & 2 \\
\hline 27 & Entropy & 0.2773 & 0.1095 & 0.3646 & 0.1065 & 0.571 & 3 \\
\hline 31 & Inf. meas. correlation & 0.0314 & 0.0189 & 0.0214 & 0.0120 & 0.448 & 14 \\
\hline \multicolumn{8}{|c|}{ Neighborhood Gray Tone Difference Matrix (NGTDM) } \\
\hline 36 & Coarseness & 9.265 & 8.236 & 21.354 & 14.909 & 0.710 & 1 \\
\hline \multicolumn{8}{|c|}{ Statistical Feature Matrix (SFM) } \\
\hline 43 & Periodicity & 0.578 & 0.081 & 0.624 & 0.064 & 0.452 & 13 \\
\hline 44 & Roughness & 2.385 & 0.127 & 2.301 & 0.100 & 0.527 & 8 \\
\hline \multicolumn{8}{|c|}{ Fractal Dimension Texture Analysis (FDTA) } \\
\hline 51 & $\mathrm{H} 1$ & 0.367 & 0.081 & 0.423 & 0.068 & 0.531 & 7 \\
\hline 52 & $\mathrm{H} 2$ & 0.291 & 0.062 & 0.336 & 0.059 & 0.521 & 9 \\
\hline
\end{tabular}

when the different feature sets are not correlated with each other and the classification results are independent. This was not always the case with the features used in this paper where some of the computed features captured complementary textural properties.

In this paper, a modular neural network system composed of ten SOM classifiers was developed. The ten different SOM classifiers were trained and evaluated using as input one of the feature sets given in Section IV. The ten classification results were combined using: 1) majority voting where the input pattern was assigned to the class with the greatest number of votes; 2) with weighted averaging based on the confidence measure. In this case, the final classification result was the average of the ten confidence measures computed as described in (3). If the final result value was negative, then the plaque was classified as symptomatic, otherwise if it was positive as asymptomatic. The confidence measure decided the contribution of each feature set to the final result. The same modular system was implemented using the statistical KNN classifier.

\section{RESULTS}

A total of 230 (115 symptomatic and 115 asymptomatic) ultrasound images of carotid atherosclerotic plaques were analyzed. Ten different texture feature sets and shape parameters (a total of 61 features) were extracted from the manually segmented plaque images as described in Section IV. For each feature set, the mean and standard deviation for the symptomatic and asymptomatic classes were computed, as well as the distance [as given in (1)] between the two classes. Unfortunately, a high degree of overlap between the values of the two classes was obtained. Best texture features using the distance given by (1) as criterion, were found to be: the coarseness of NGTDM, the entropy, the mean and range of values of the angular second moment, and entropy of SGLDM, the fractal values $\mathrm{H} 1$ and $\mathrm{H} 2$, the median gray level value, and the roughness and the periodicity of SFM. Table I tabulates the results for the 15 best feature sets. In general, texture in symptomatic plaques tends to be more dark, with higher contrast, more heterogeneous, more rough and less periodical, whereas in asymptomatic plaques texture tends to be brighter, with less contrast, more homogeneous, more smooth, with large areas with small gray tone variations, and more periodical. This confirms the original assumption [2]-[7] that smooth surface, echogenicity, and a homogenous texture are characteristics of stable plaques, whereas irregular surface, echolucency and a heterogeneous texture are characteristics of potentially unstable plaques. Table II summarizes the texture characteristics of symptomatic Vs asymptomatic plaques as these were obtained by interpretation of the values of the texture features given in Table I. Fig. 5 displays 2-D plots of combinations of some of the first best features tabulated in Table I. The figure shows the high degree of overlap of the texture features for the two classes and the difficulty of the problem.

For the classification task, the neural SOM classifier and the statistical KNN classifier were implemented. For training the classifier, 80 symptomatic and 80 asymptomatic plaques were used, whereas for evaluation of the system the remaining 35 
TABLE II

Texture Characteristics of Symptomatic Versus Asymptomatic Plaques as TheSe Were ObTained by Interpretation of the TeXture FEATURES VALUES GIVEN IN TABLE I

\begin{tabular}{l|l}
\hline \multicolumn{1}{c|}{ Symptomatic Plaques } & \multicolumn{1}{c}{ Asymptomatic Plaques } \\
\hline more dark & brighter \\
\hline higher contrast & less contrast \\
\hline more rough & more smooth \\
\hline more heterogeneous & more homogeneous \\
\hline less periodical & more periodical \\
\hline $\begin{array}{l}\text { less coarse, i.e. less local uniformity } \\
\text { in intensity }\end{array}$ & $\begin{array}{l}\text { more coarse, i.e. large areas with } \\
\text { small gray tone variations }\end{array}$ \\
\hline
\end{tabular}
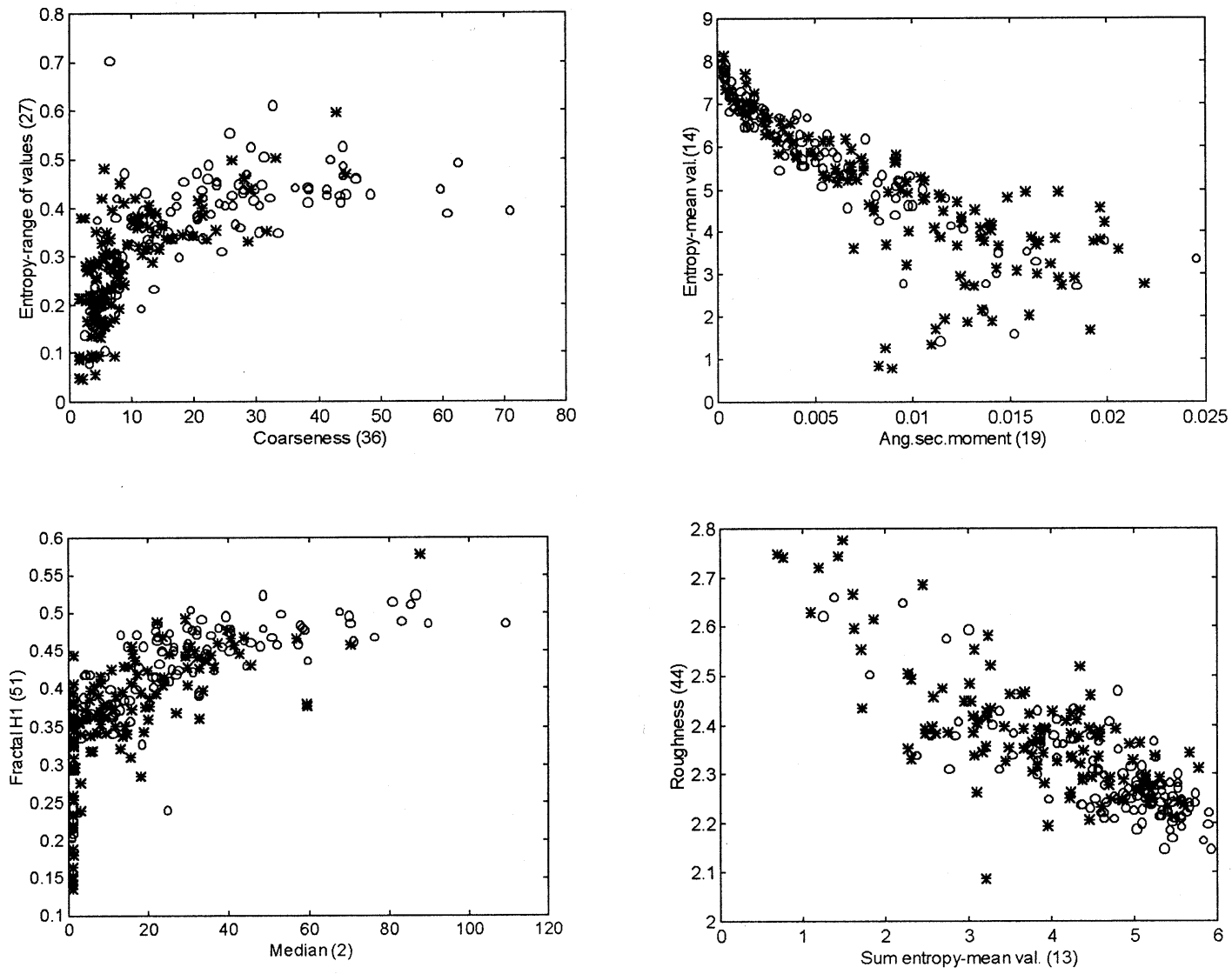

Fig. 5. Two dimensional plots for the 230 carotid plaques, of several combinations of some of the best features as these are tabulated in Table I $(*=$ symptomatic, $\mathrm{o}=$ asymptomatic). The numbers in brackets denote the serial feature number as listed in Table I. All plots show the high degree of overlap between the symptomatic and asymptomatic plaque classes.

symptomatic and 35 asymptomatic plaques were used. In order to verify the correctness of the classification results, a bootstrapping procedure was followed. The system was trained and evaluated using five different bootstrap sets where in each set 160 different plaques were selected at random for training and 70 different plaques for evaluation. Table III tabulates the average diagnostic yield (DY) obtained for the two classifiers, for the ten different feature sets, and when the classification results were combined. The DY is defined as the percentage of the correctly classified plaques to the total number of the tested plaques.

The unsupervised neural SOM classifier was implemented with a $10 \times 10$ output node architecture and it was trained for 5000 learning epochs. Different neighborhood window sizes were evaluated for computing the confidence measure, where the $5 \times 5$ window size proved to give in average the best results. Best feature set was the SGLDM (range values) with 70.0\%, followed by the NGTDM with 68.3\%, the Laws TEM with 67.4\%, and the GLDS with $66.8 \%$. Worst feature set was the shape parameters with DY only $52.8 \%$, which means that they contain little diagnostic information. The combination of the classification results improved the average DY for the ten feature sets from $64.9 \%$ up to $66.0 \%$ when combined with majority voting, and up to $73.1 \%$ when combined with the confidence measure. Fig. 4 illustrates the distribution of 160 carotid plaques of the training set on a $10 \times 10 \mathrm{SOM}$, using as input the SGLDM (range values) feature set that gave in average the highest DY. In addition, the DY was computed using all 61 features as one feature set, which yielded $68.8 \%$, and for the 15 best features that 
TABLE III

Mean and Standard DeViation Of The DY, for the Evaluation SET, For Five Bootstrap Sets, For the SOM Classifier For a $5 \times 5$ Neighborhood

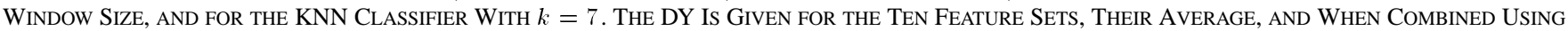
Majority Voting and by Averaging the Ten Confidence Measures. Also, the DY Is Given for All the 61 Features Used as One Feature Set, AND FOR THE 15 Best Features AS Selected Using (1) AND SHOWN IN TABle I

\begin{tabular}{c|c|c|c}
\hline Feature Set & $\begin{array}{c}\text { Feature Set } \\
\text { Vector Size }\end{array}$ & $\begin{array}{c}\text { SOM } \\
\text { Classifier } \\
\text { DY\% }\end{array}$ & $\begin{array}{c}\text { KNN Classifier } \\
\text { DY\% }\end{array}$ \\
\hline FOS & 5 & $65.7 \pm 3.9$ & $61.7 \pm 5.1$ \\
\hline SGLDM (mean) & 13 & $66.2 \pm 4.6$ & $66.5 \pm 3.3$ \\
\hline SGLDM (range) & 13 & $70.0 \pm 1.5$ & $67.1 \pm 3.7$ \\
\hline GLDS & 4 & $66.8 \pm 3.1$ & $64.0 \pm 4.4$ \\
\hline NGTDM & 5 & $68.3 \pm 3.4$ & $62.8 \pm 3.7$ \\
\hline SFM & 4 & $65.1 \pm 4.4$ & $59.7 \pm 5.1$ \\
\hline TEM & 6 & $67.4 \pm 5.6$ & $63.4 \pm 7.0$ \\
\hline FDTA & 4 & $63.7 \pm 6.8$ & $64.9 \pm 2.1$ \\
\hline FPS & 2 & $62.6 \pm 6.8$ & $59.7 \pm 2.4$ \\
\hline Shape parameters & 5 & $52.8 \pm 6.3$ & $55.7 \pm 7.1$ \\
\hline Average & & $64.9 \pm 4.7$ & $62.5 \pm 4.4$ \\
\hline $\begin{array}{c}\text { Combine the ten feature } \\
\text { sets } \text { with majority voting }\end{array}$ & & & \\
\hline $\begin{array}{c}\text { Combine by averaging the } \\
\text { ten confidence measures }\end{array}$ & & $66.0 \pm 2.8$ & $65.1 \pm 3.3$ \\
\hline & & $73.1 \pm 4.2$ & $68.8 \pm 3.9$ \\
\hline All 61 features & 61 & $68.8 \pm 2.2$ & $69.7 \pm 2.3$ \\
\hline 15 best features & 15 & $68.0 \pm 5.4$ & $66.9 \pm 4.2$ \\
\hline
\end{tabular}
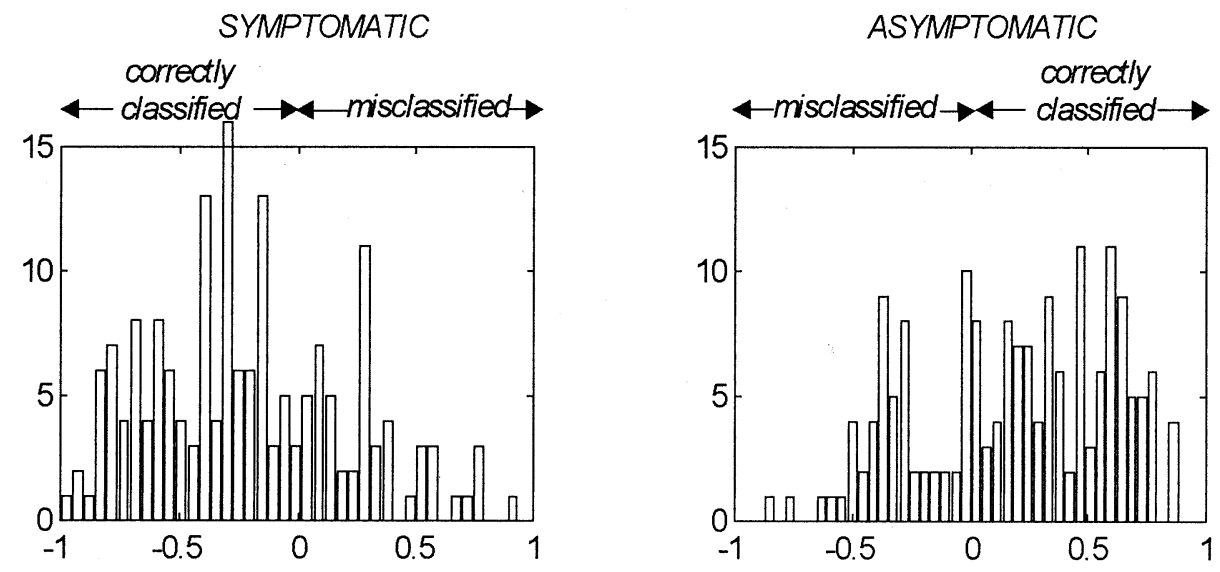

Fig. 6. Histograms of the distribution of the average confidence measure for the five bootstraps, for the symptomatic and asymptomatic cases, for the SOM system using as input the SGLDM (range) feature set. For the symptomatic cases negative values indicate the correctly classified plaques whereas the positive values indicate the misclassified plaques. The reverse applies for the asymptomatic cases. Values close to zero mean low confidence.

were selected as described in (1), which yielded $68.0 \%$. These results were better than the average DY of the individual feature sets but worse than the overall DY of the combiner. These results are also tabulated in Table I.

For comparison reasons the statistical KNN classifier was also implemented, which also performed comparably well. In Table III, the results are tabulated for $k=7$ that gave the highest combined DY. This was $68.8 \%$ and it was obtained when combining the results by averaging the ten confidence measures. The DY when combining the results with simple majority voting was $65.1 \%$. The best individual result for the KNN classifier was also achieved with the SGLDM (range) feature set, which was $67.1 \%$, followed by the SGLDM (mean) with a DY of $66.5 \%$, the FDTA with a DY of $64.9 \%$, and the GLDS with a DY of $64.0 \%$. These feature sets performed well also with the SOM system. Best results for the KNN system were obtained when all 61 features were used as one feature set, with $69.7 \%$.

Fig. 6 shows two histograms of the distribution of the combined average confidence measure for the five bootstrap sets, for the symptomatic and asymptomatic cases using the SOM classifier and the SGLDM (range) feature set. For the symptomatic cases negative values indicate the correctly classified plaques whereas the positive values indicate the misclassified plaques. The reverse applies for the asymptomatic cases. The value of the average confidence measure indicates the degree of confidence of the final classification result. Values close to -1 or close to 1 mean high confidence, whereas values close to 0 mean low confidence.

Receiver operating characteristic (ROC) analysis is a standard approach to evaluate the sensitivity and specificity of 


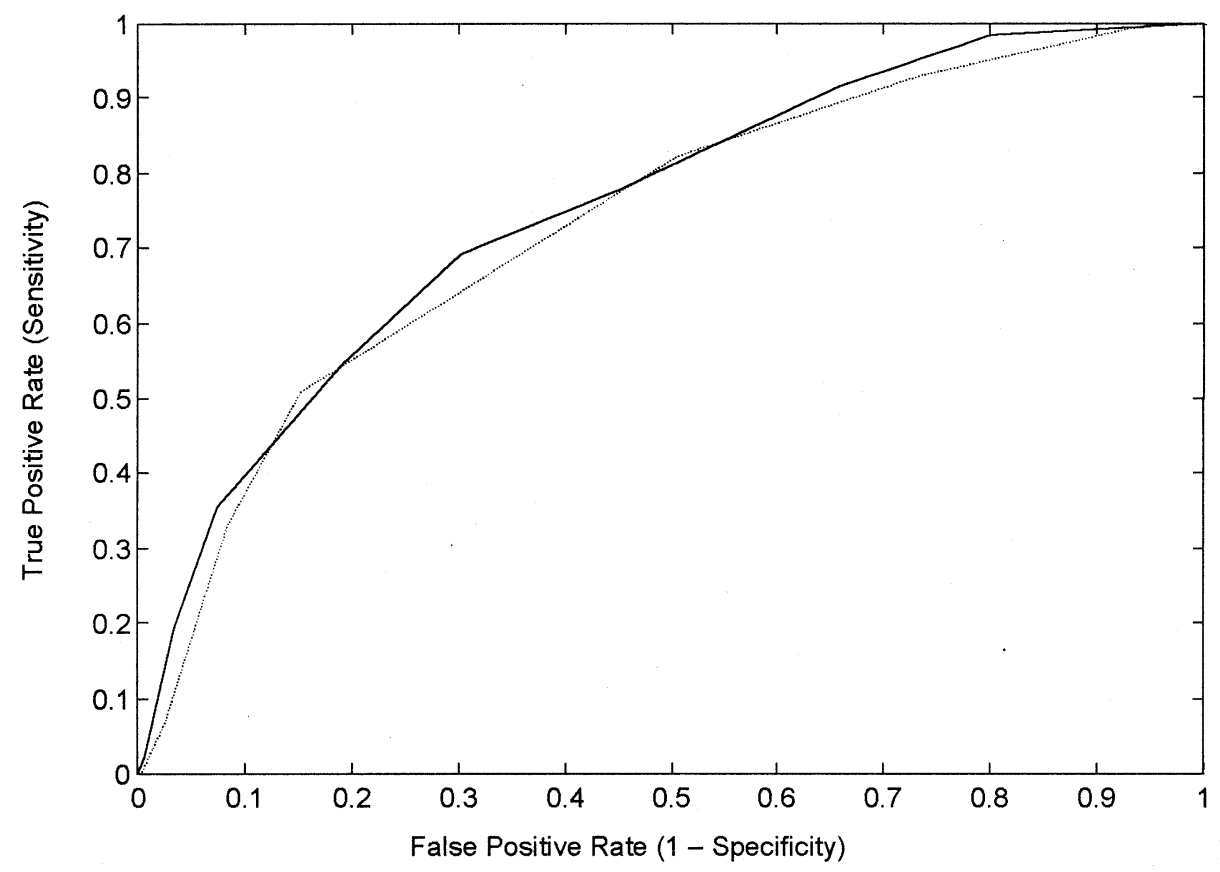

Fig. 7. ROC analysis based on the distributions of the average confidence measure as shown in Fig. 6, for the SOM and the KNN (dotted curve) classifiers, using as input the SGLDM (range) feature set. The area below the curve is for the SOM classifier 0.753 whereas for the KNN classifier is 0.738 .

diagnostic procedures [25]. ROC analysis estimates a curve of the true positive rate (sensitivity) Vs the false positive rate ( 1 - specificity), which describes the inherent tradeoff between sensitivity and specificity of a diagnostic system. The area below the curve indicates the discrimination capability of the specific system. Fig. 7 shows the ROC curves for the SOM and the KNN classifiers, for the SGLDM (range) feature set, based on the distributions of the average confidence measure as shown in Fig. 6. The area below the curve was slightly higher for the SOM classifier, 0.753, whereas for the KNN classifier was 0.738 .

\section{DISCUSSION}

In this study, a modular system using multiple texture features and multiple classifiers is proposed for the classification of carotid plaques. Such a system will help in enhancing the significance of noninvasive cerebrovascular tests in the identification of asymptomatic carotid stenosis at risk of stroke. A total of 61 texture features and shape parameters were extracted from the ultrasonic carotid plaque images. The statistics for all the texture features extracted indicate a high degree of overlap between the symptomatic and asymptomatic groups. This makes the separation and the classification tasks of the two groups very difficult.

In previous work, a relationship between plaque morphology and the risk of stroke was reported [2]-[7]. El-Barghouty et al. [3] used the GSM of the ultrasound plaque image for the characterization of plaques as echolucent $(\mathrm{GSM} \leq 32)$ and echogenic (GSM > 32). Elatrozy et al. [5] reported that plaques with GSM $<40$ are good predictors of ipsilateral hemispheric symptoms related to carotid plaques. In this paper, the cutoff GSM value was found to be about 23 (GSM $\leq 23$ for symptomatic plaques and GSM $>23$ for asymptomatic plaques). The difference in the GSM value computed in this paper, compared with that of El-Barghouty et al. [3] can be attributed to the fact that the gray level of the plaque images was not standardized using blood and adventitia as reference. In the case of Elatrozy et al. [5], a standardization procedure was followed, as used in this paper, but with different values for the reference points. Furthermore, the use in this study of a color image indicating the blood flow for segmenting the plaque, enabled the more correct outline of the plaque region. This was especially true in the case of highly echolucent (dark) plaques where the plaque boundaries were not otherwise visible and, therefore, dark areas of the plaque could have been omitted. This can explain why the GSM value of 23 reported in this paper is lower than the GSM values reported in the other two studies.

In most of the above studies [2]-[7], the characteristics of the plaques were usually subjectively defined or using simple statistical measures, and the association with symptoms was established through simple statistical analysis. In this paper, a large number of texture features were extracted directly from the plaque ultrasound images, and they were used in order to build an automated system, which can classify carotid plaques as symptomatic or asymptomatic, based on a multifeature multiclassifier paradigm.

Standardization using blood and adventitia as reference points, is an important preprocessing step, which affects the quality of the results. As previous work has shown [8], images obtained from different scanners, by different ultrasonographers and through different peripherals, can be standardized so that measurements of the plaque echodensity may become comparable. This step is necessary in order to get images with low intra- and interobserver variability, which will allow the development of meaningful studies in relation to clinical events.

The neural SOM classifier and the statistical KNN classifier were implemented for the classification of the carotid plaques. 
The SOM was chosen because it is an unsupervised learning paradigm where the input patterns are freely distributed over the output node matrix, allowing an efficient mapping of the input data with no need to create exact classification boundaries. The SOM is well suited for pattern classification problems, where there is a high degree of overlap between the classes as this was the case in this study. Ten different texture and shape feature sets were extracted from the plaque images and used for training multiple SOM classifiers. Best feature sets were the SGLDM (range) feature set, followed by the NGTDM, the Laws TEM, and the GLDS. In general, all feature sets performed in a range of about $62 \%-70 \%$, except of the shape parameters that performed much worse.

In order to enhance the classification success rate, the classification results of the ten feature sets were combined, by averaging the confidence measure derived from the SOM classifier. The confidence measure decided the contribution of each feature set to the final classification result. Combining the classification results of the ten different feature sets with the use of a confidence measure, improved the classification results obtained by the individual feature sets, reaching an average DY of $73.1 \%$ for the evaluation set. The benefits of combining are more obvious in the case where there are no dominant best feature sets or best classifiers, as was the case with the features extracted from the carotid plaque images in this study. Combining and averaging of classifiers can be beneficial when there is no reliable method for selecting the best classifier or when several classifiers have similar performance [23]. The idea behind combining is that although one of the classifiers will eventually yield the best performance, the sets of patterns misclassified by the different classifiers using as input the different feature sets, will not necessarily overlap. This suggests that different classifier designs potentially offer complementary information, which could be harnessed to improve the overall classifier performance [24].

The statistical KNN classifier was also implemented for the classification of carotid plaques, which also performed well and yielded results comparable in most cases with the results obtained by the SOM classifier. The best individual result for the KNN classifier was also achieved with the SGLDM (range) feature set, with a DY of $67.1 \%$. The KNN classifier was computationally much faster compared to the training time needed by the SOM classifier, which was about 1 minute on a PC Pentium 4 at $1.5 \mathrm{GHz}$. The optimal choice of $k$ entails a tradeoff between bias (accuracy) and variance (precision). Likewise, the dimensionality of the SOM entails a similar tradeoff between bias and variance. In this paper, the $k$ value and the SOM size were so chosen so that to yield the highest classification score.

The confidence measure computed as given in (4) is a qualitative measure of the degree a plaque belongs to the assigned class. Fig. 6 illustrates the histograms of the confidence measure distribution of the plaques analyzed in this paper. The range of the confidence measure is from -1 (symptomatic) to 1 (asymptomatic), where values close to 0 mean low confidence. The in-between values indicate the degree a plaque can be characterized as symptomatic or asymptomatic. In a prospective study where the patients could be followed up and assessed over a pe- riod of time, it will be interesting to investigate how this qualitative measure is affected by medication or other treatment.

The quality of the ultrasound images affects the quality of the extracted texture features. As the quality of the ultrasound devices improves, it will be possible to acquire better quality ultrasound images, which will improve the system performance. Another way to improve performance will be through a threedimensional (3-D) reconstruction of the plaque, which will allow the extraction of global 3-D texture features characterizing the carotid plaque [26]. Such 3-D information, not considered in 2-D features will provide a better representation of the plaque structure and when used for classification will contribute toward a higher DY. Furthermore, additional sources of information like clinical data, risk factors and stroke related laboratory factors may be integrated into a hybrid diagnostic system. The full automation of the system will be also the subject of future work where the automation of the carotid plaque identification and segmentation, and the image standardization will be investigated. This will reduce any observer biases concerning the location and size of the plaque.

In conclusion, the results in this paper show that it is possible to identify a group of patients at risk of stroke based on texture features extracted from high-resolution ultrasound images of carotid plaques. This group of patients may benefit from a carotid endarterectomy whereas other patients may be spared from an unnecessary operation. Because of the difficulty of the problem and the high degree of overlap of the symptomatic and asymptomatic classes, the above results should be verified with more images from more patients.

\section{ACKNOWLEDGMENT}

This work was part of a European Union project (Biomed 2 Program-PL 950629) carried out in centers all over Europe and coordinated by the St. Marys Hospital, London, U.K. The aim of the project was to evaluate the value of noninvasive investigations in the identification of individuals with asymptomatic carotid stenosis at risk of stroke (ACSRS) [1].

\section{REFERENCES}

[1] A. Nicolaides, "Asymptomatic carotid stenosis and the risk of stroke (The ACSRS study): Identification of a high risk group," in Cerebrovascular Ischaemia Investigation and Management. Los Angeles, CA: Med-Orion, 1996, ch. 38, pp. 435-441.

[2] G. Geroulakos, J. Domjan, A. Nicolaides, J. Stevens, N. Labropoulos, G. Ramaswami, and G. Belcaro, "Ultrasonic carotid artery plaque structure and the risk of cerebral infraction on computed tomography," J. Vasc. Surg., vol. 20, no. 2, pp. 263-266, August 1994.

[3] N. El-Barghouty, G. Geroulakos, A. Nicolaides, A. Androulakis, and V. Bahal, "Computer assisted carotid plaque characterization," Eur. J. Vasc. Endovasc. Surg., vol. 9, pp. 548-557, 1995.

[4] A. Iannuzzi, T. Wilcosky, M. Mercury, P. Rubba, F. Bryan, and G. Bond, "Ultrasonografic correlates of carotid atherosclerosis in transient ischemic attack and stroke," Stroke, vol. 26, no. 4, pp. 614-619, April 1995.

[5] T. Elatrozy, A. Nicolaides, T. Tegos, and M. Griffin, "The objective characterization of ultrasonic carotid plaque features," Eur. J. Vasc. Endovasc. Surg., vol. 16, pp. 223-230, 1998.

[6] J. E. Wilhjelm, L. M. Gronholdt, B. Wiebe, S. K. Jespersen, L. K. Hansen, and H. Sillesen, "Quantitative analysis of ultrasound B-mode images of carotid atherosclerotic plaque: Correlation with visual classification and histological examination," IEEE Trans. Med. Imag., vol. 17, pp. 910-922, Dec. 1998. 
[7] J. Polak, L. Shemanski, D. O'Leary, D. Lefkowitz, T. Price, P. Savage, W. Brand, and C. Reld, "Hypoechoic plaque at US of the carotid artery: An independent risk factor for incident stroke in adults aged 65 years or older," Radiology, vol. 208, no. 3, pp. 649-654, Sept. 1998.

[8] T. Elatrozy, A. Nicolaides, T. Tegos, A. Zarka, M. Griffin, and M. Sabetai, "The effect of B-mode ultrasonic image standardization on the echogenicity of symptomatic and asymptomatic carotid bifurcation plaques," Int. Angiol., vol. 17, no. 3, pp. 179-186, September 1998.

[9] M. Amadasun and R. King, "Textural features corresponding to textural properties," IEEE Trans. Syst., Man, Cybern., vol. 19, pp. 1264, 269-1274, 285, Sept./Oct. 1989.

[10] J. S. Weszka, C. R. Dyer, and A. Rosenfield, "A comparative study of texture measures for terrain classification," IEEE Trans. Syst., Man, Cybern., vol. SMC-6, Apr. 1976.

[11] C.-M. Wu, Y.-C. Chen, and K.-S. Hsieh, "Texture features for classification of ultrasonic liver images," IEEE Trans. Med. Imag., vol. 11, pp. 141-152, June 1992

[12] Y. M. Kadah, A. A. Farag, J. M. Zurada, A. M. Badawi, and A. M. Joussef, "Classification algorithms for quantitative tissue characterization of diffuse liver disease from ultrasound liver images," IEEE Trans. Med. Imag., vol. 15, pp. 466-478, Aug. 1996.

[13] T. Ojala, M. Pietikainen, and D. Harwood, "A comparative study of texture measures with classification based on feature distributions," Pattern Recogn., vol. 29, no. 1, pp. 51-59, 1996.

[14] W. H. Press, B. P. Flannery, S. A. Teukolsky, and W. T. Vetterling, Numerical Recipes, The Art of Scientific Computing. Cambridge, U.K.: Cambridge Univ. Press, 1987.

[15] R. M. Haralick, K. Shanmugam, and I. Dinstein, "Texture features for image classification," IEEE Trans. Syst., Man, Cybern., vol. SMC-3, pp. 610-621, Nov. 1973
[16] C.-M. Wu and Y.-C. Chen, "Statistical feature matrix for texture analysis," CVGIP: Graphical Models Image Processing, vol. 54, no. 5, pp. 407-419, September 1992.

[17] K. I. Laws, "Rapid texture identification," Proc. SPIE, vol. 238, pp. $376-380,1980$

[18] B. B. Mandelbrot, The Fractal Geometry of Nature. San Francisco, CA: Freeman, 1982.

[19] S. Theodoridis and K. Koutroumbas, Pattern Recognition. New York: Academic, 1999.

[20] C. I. Christodoulou, C. S. Pattichis, M. Pantziaris, T. Tegos, A. Nicolaides, T. Elatrozy, M. Sabetai, and S. Dhanjil, "Multi-feature texture analysis for the classification of carotid plaques," presented at the Int. Joint Conf. Neural Networks IJCNN '99, Washington DC, July 10-16, 1999.

[21] T. Kohonen, "The self-organizing map," Proc. IEEE, vol. 78, no. 9, pp. 1464-1480, Sept. 1990.

[22] J. T. Tou and R. C. Gonzalez, Pattern Recognition Principles. Reading, MA: Addison-Wesley, 1974

[23] M. P. Perrone, "Averaging/modular techniques for neural networks," in The Handbook of Brain Theory and Neural Networks, M. A. Arbib, Ed. Cambridge, MA: MIT Press, 1995, pp. 126-129.

[24] J. Kittler, M. Hatef, R. Duin, and J. Matas, "On combining classifiers," IEEE Trans. Pattern Anal. Machine Intell., vol. 20, pp. 226-239, Mar. 1998.

[25] J. A. Swets and R. M. Pickett, Evaluation of Diagnostic Systems: Methods from Signal Detection Theory. New York: Academic, 1992.

[26] V. Kovalev and M. Petrou, "Texture analysis in three dimensions as a cue to medical diagnosis," in Handbook of Medical Imaging_Processing and Analysis, I. N. Bankman, Ed. New York: Academic, 2000, pp. 231-247. 